\title{
The application of Bayesian filter and neural networks in lane changing
}

\section{prediction}

\author{
LI Li ${ }^{1, a^{*}}$, ZHANG Mingfang ${ }^{1, b}$, LIU Rui ${ }^{2, c}$ \\ 1Chang'an University, School of Automobile, Xi'an 710064, China \\ 2Shenzhen Expressway Company Limited, Shenzhen 518000, China \\ a991958405@qq.com,b57631035@qq.com, ${ }^{\text {a } 593143197 @ q q . c o m ~}$
}

Keyword: Lane change intent; BP neural network; Bayesian filter; Prediction; Correction

Abstract: In order to improve safety during lane change, we proposed lane change intent prediction method based on neural networks and Bayesian filters. The method uses the lane line sensor, steering wheel angle sensor and in-vehicle CAN bus acquisition characterization parameters. The above acquisition parameters as the neural network input data, driver's lane change intention preliminary forecast, take the output of BP neural network as the input of Bayesian filters, and then amendments the results of BP neural network. Using real vehicle lane changing data training and testing the model. The results show that prediction accuracy rate of BP neural network and Bayesian filters reaches $91.38 \%$. The forecast accuracy increased by 6 percentage points compare to single BP neural network and has better versatility.

\section{Introduction}

In order to prevent the collision between vehicle and around the vehicle in the lane change process, people developed a lot of driver assistance systems, like lane change warning systems. These systems obtain the relative motion relationship between own vehicle and the target vehicle in real-time status, but they cannot predict the lane changing of own vehicle. When there is potential hazardous situation, the driver's reaction time is too short .so the systems can't reach the advanced active safety system requirements. Therefore, the predicting of the intent of the driver to change lanes has practical application value.

Currently, about all the research on the driver intention to change the lane, the representative results are as follows: Koji Suzuki ${ }^{[1]}$ use artificial neural network established own vehicle lane change intent forecast model based on the car position and movement relationship between own vehicle and the target vehicle. According to the heading angle of the own car and the distance between lane line, based motion model and operation recognition Adam Houenou ${ }^{[2]}$ established a predictive model to predict the vehicle trajectory. Yi Hou ${ }^{[3]}$ established a lane change intent forecasting method based on Bayesian filter and decision tree. The domestic researches in related fields are mainly in universities, and we have a lot of literature who predict the lane change intention based on the characteristics of eye movement ${ }^{[4-6]}$. In addition, Peng Jinshuan tied into account the factors of both the driver and the vehicle, and combined the driver and vehicle factors to identify the drivers' intention to change lanes, with high recognition accuracy. Similarly, HOU Haijing of Jilin University proposed method to recognize lane changing behavior characteristics of the driver on the highway .etc.

All in all, the current research focused on the prediction of the lane change intention is mainly in foreign .And methods used are based on large-car sensor parameters, and then establish relevant 
predictive models. Foreign research methods may use too many arguments, or establish complex predictive model. They cannot meet the needs that the active safety system should deal with problems quickly. For this, we proposed a method to predict the drivers' lane-changing intention based on the neural network and Bayesian filter. We use lane line sensor, steering wheel angle sensor and vehicle CAN bus to obtain the original parameters. Then predict by the neural network initially, and the output results are the input data of Bayesian filters to correct the predicted results. At last, we obtained the final prediction results. Lane change intention forecasting process is shown in Figure 1.

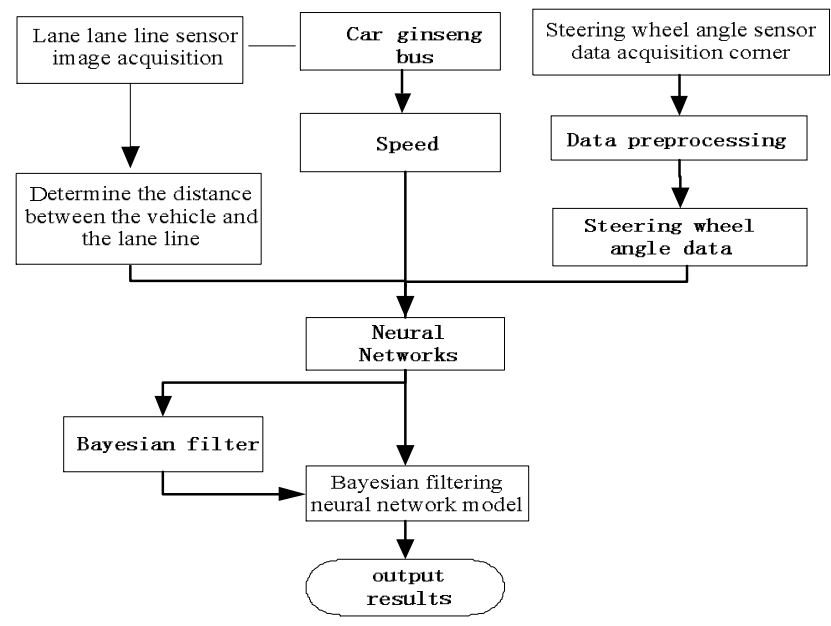

Figure 1. Lane change intention forecasting process

\section{Lane change intention forecast by BP neural network and Bayesian filters}

The selection of characterizing parameter. (1)Distance $d$ between the vehicle and the lane markings. The distance between the vehicle and lane markings is the most direct manifestation of lane change form. The distance between the vehicle and lane markings will gradually decrease in the lane change process, and then gradually increase.

(2) Steering wheel angle $\theta$.In the lane change process drivers choose steering operation inevitably. The steering wheel angle reflects the operational drivers' behavior to change lanes for a certain extent. Compared with torque in the process of changing lanes, there are certain differences.

(3) Speed $v$. Lane-changing behavior is a process integrated drivers' demand and state of the vehicles' relative motion. In the course of lane change the speed presents certain acceleration or deceleration law.

Training samples and test samples. In this article the training and testing samples come from the real vehicle data, and the data is collected in Xi'an beltway. We selected 735 groups of vehicle traveling state data, lane keeping and lane-changing are 423 groups and 312 groups. Portion of the selected data used to train the model, part of data used for testing the model. Training and testing samples were 492 groups and 243 groups. Data ratio of lane change and lane keeping are shown in Figure 2, 3. 


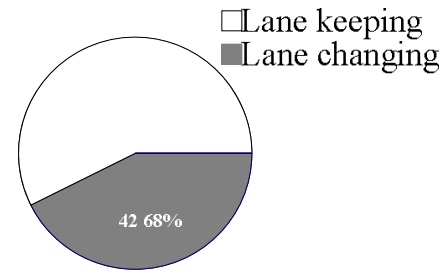

Figure2. Training sample composition

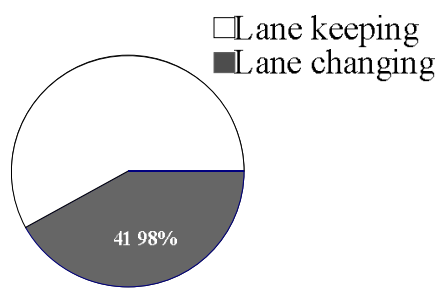

Figure 3. Prediction sample composition

Model Training. This article is intended for the driver to predict the intention of lane change in real time, and the collected raw data changed over time sequence. So the timing window is particularly important. On the one hand, if the time window is too long, the data processing will be longer. While the data processing time is too long, the sampling frequency will be reduced, and the forecast accuracy will be declined. It is not conducive to real-time processing model. On the other hand, if the time window is too short data law will not be obvious, and false alarm rate of the system will increase. In this regard, we use the model's accuracy of prediction as the evaluation index of the time window length. The model prediction accuracy obtained at different time window length is shown in Figure4.

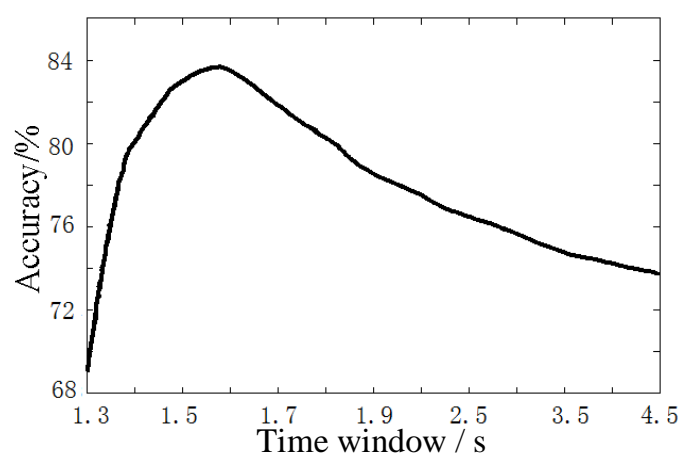

Figure 4 the determination of the time window

As can be seen from Figure 4, when the time window length is 1.6 seconds, the prediction accuracy of the model is the highest. So 1.6 seconds is the final choice of the time window length.

The sample number in the model train is 492, of which lane change and lane keeping accounting are respectively $42.68 \%$ and $57.32 \%$. Training results expressed numerically, and the specific figures indicate the state of motion type relationship of the vehicle are shown in Table 1.

Table 1 vehicle motion state number

\begin{tabular}{ccc}
\hline $\begin{array}{c}\text { Vehicle } \\
\text { motion }\end{array}$ & $\begin{array}{c}\text { Lane } \\
\text { changing }\end{array}$ & Lane keeping \\
\hline Number & 1 & 0 \\
\hline
\end{tabular}

When training the model, the input of the BP neural network are lane-keeping and lane-changing behavior characterization parameters, and output the corresponding results, making BP neural network model to tap the intrinsic link between input and output. The accuracy of the single neural network training is $85.4 \%$. According to the model design, and then the output of BP neural network is transmitted to the Bayesian filter. Bayesian filter estimated motion state from the car stage firstly, combined with the output of BP neural network, and then the final output is given.

Part of the obtained results outputted from different types of models are shown in Table 2, where $\mathrm{BF}$ represents the prediction of Bayesian filter, $\mathrm{BP}+\mathrm{BF}$ represents a comprehensive forecast results of neural networks and Bayesian filter.

In this case, the prediction accuracy of neural networks and Bayesian filter model was 
91.38\%. Compared with a single BP neural network, this model has a higher prediction accuracy.

Table 2 Model prediction and correction results

\begin{tabular}{|c|c|c|c|c|c|}
\hline BP neural prediction results & 1 & 1 & 1 & 1 & 1 \\
\hline BF prediction results & $\begin{array}{c}0.6 \\
1 \\
\end{array}$ & 0.33 & 0.59 & 0.37 & 0.63 \\
\hline $\mathrm{BP}+\mathrm{BF}$ results & 1 & 0 & 1 & 0 & 1 \\
\hline The true value & 1 & 0 & 1 & 0 & 1 \\
\hline BP neural prediction results & 1 & 0 & 1 & 0 & 1 \\
\hline BF prediction results & $\begin{array}{c}0.6 \\
1\end{array}$ & 0.33 & 0.58 & 0.46 & 0.55 \\
\hline $\mathrm{BP}+\mathrm{BF}$ results & 1 & 0 & 1 & 0 & 1 \\
\hline The true value & 1 & 0 & 1 & 0 & 1 \\
\hline
\end{tabular}

\section{Conclusion}

Based on the good predictive ability of BP neural network model and Bayesian theory, combined with the demand of prediction beforehand of the vehicle active safety system, we proposed lane change intent prediction method based on BP neural networks and Bayesian filters. And through the model we achieve the intention to change lanes. Through these studies we have the following conclusions:

1. The time window has an important impact on the accuracy of the prediction model by testing different prediction accuracy of different time window length, finally we select $1.5 \mathrm{~s}$ as the length of the time window.

2. Using a single BP neural network for prediction, the prediction accuracy is $85.4 \%$. While use of BP neural networks and Bayesian filter, the model prediction accuracy is $91.38 \%$.

3. Through analyzing ROC curve of single BP neural network model, BP neural networks and Bayesian filter model, it is suggested that the latter has a broader versatility.

\section{Reference}

[1] Zheng J, Suzuki K, Fujita M. Predicting driver's lane-changing decisions using a neural network model[J]. Simulation Modelling Practice and Theory, 2014, 42: 73-83.

[2] Houenou A, Bonnifait P, Cherfaoui V, et al. Vehicle trajectory prediction based on motion model and maneuver recognition[C]//Intelligent Robots and Systems (IROS), 2013 IEEE/RSJ International Conference on. IEEE, 2013: 4363-4369.

[3] Hou Y, Edara P, Sun C. Modeling Mandatory Lane ChangingUsing Bayes Classifier and Decision Trees [J]. IEEE Transactions on Intelligent Transportation Systems. 2014, 15(2): 647-655.

[4] Wei Yuan, Rui Fu, Yingshi Guo, et al. Driver's lane changing intention identification based on visual characteristics [J]. China Journal of Highway and Transport, 2013,26(4):132-138(in Chinese).

[5] Cun Sun. Based on driver's visual characters lane change intention recognition [D]. Xi'an: Chang'an University, 2012: 26-45. (in Chinese).

[6] Jin-shuang Peng, Rui Fu, Ying-shi Guo, et al. Lane change intent identification based on drivers' visual characteristics [J]. Journal of Wuhan University of Technology, 2013, 35(3): 73-79 (in Chinese). 